\title{
A Step Towards Change: A Green Alternative for the Synthesis of Metal-Organic Frameworks
}

\author{
Paola Marino, ${ }^{\text {ab }}$ P. Rafael Donnarumma, ${ }^{\text {ab }}$ Hudson de Aguiar Bicalho, ${ }^{\text {ab }}$ \\ Victor Quezada-Novoa, ${ }^{\text {ab }}$ Hatem M. Titic and Ashlee J. Howarth ${ }^{*}$ ab \\ a. Department of Chemistry and Biochemistry, and Centre for NanoScience Research, \\ Concordia University, 7141 Sherbrooke St. W., Montreal, Quebec, H4B 1R6, Canada. E-mail: ashlee.howarth@concordia.ca \\ b. FRQNT - Quebec Centre for Advanced Materials, Montreal, Quebec, Canada. \\ c. Department of Chemistry, McGill University, 801 Sherbrooke St. W., Montreal, Quebec, H3A OB8, Canada.
}

\begin{abstract}
Metal-organic frameworks (MOFs) are traditionally synthesized using formamide solvents that are hazardous to human health and the environment. In order to alleviate the environmental impact of MOF synthesis in both academic and industrial settings, safer and greener solvent alternatives are desired. Herein, STEPOSOL ${ }^{\circledR}$ MET-10U ( $N, N$-dimethyl-9-decenamide), a bioderived solvent produced via olefin metathesis using renewable feedstocks, such as plant oils, is explored as a solvent for the synthesis of a series of structurally diverse MOFs.
\end{abstract}

\section{Introduction}

The development and implementation of green synthetic procedures is important for minimizing the environmental impact of industrial and academic research in the chemical sciences. ${ }^{1}$ Our environment is suffering considerably due to many global issues including, but not limited to, waste disposal, natural resource depletion, and air, water and soil pollution. ${ }^{2}$ The comparatively new field of chemistry, green chemistry, embraces and implements the notion of developing chemical products and processes that seek to mitigate and/or eliminate the use/generation of hazardous substances. ${ }^{1 a}$ More specifically, less hazardous chemical synthesis (principle \#3), safer solvents and auxiliaries (principle \#5), and renewable feedstocks (principle \#7) are three principles of green chemistry that encourage the discovery of greener alternatives in synthetic chemistry. ${ }^{1 \mathrm{c},}$ 1d

Metal-organic frameworks (MOFs) are an intriguing class of porous, and often crystalline, materials that are comprised of metal nodes bridged by organic linkers, giving rise to network materials. $^{3}$ The notable designability of MOFs, coupled with high surface area and porosity has enabled their study in various applications such as gas capture and storage, ${ }^{4}$ catalysis, ${ }^{5}$ chemical sensing, ${ }^{6}$ wastewater remediation, ${ }^{7}$ drug delivery, ${ }^{8}$ and solar fuels generation, ${ }^{9}$ amongst others. Demonstrating that these potential applications can become a reality, the first commercial MOF products were released in 2017. ${ }^{10} \mathrm{MOFs}$ have shown great promise in the field of green chemistry, from synthesis $^{11}$ to application. ${ }^{12}$ Like most fields of chemistry however, there are still many challenges that lie ahead to ensure the sustainable synthesis and application of MOFs. ${ }^{11 c}$ Several MOFs, including those studied for potential green applications, are still synthesized in, and washed with, high boiling point and hazardous solvents such as $\mathrm{N}, \mathrm{N}$ dimethylformamide (DMF), meaning that in some cases, extensive volumes of DMF are used. Risks are therefore twofold - not only to the environment, but also to human health, where acute exposure to DMF can cause hepatotoxicity, while chronic exposure can lead to reproductive issues and cancer in humans. ${ }^{13}$ As a result, the Registration, Evaluation, Authorisation and Restriction of Chemicals (REACH) has listed DMF as a "substance of very high concern", while the Pfizer solvent selection guide lists DMF as "undesirable" for use. ${ }^{14}$ Although DMF can be used safely with proper personal protective equipment (PPE) in the laboratory, and can also be recycled in some instances, finding greener alternatives to $D M F$ is of interest.

Herein, we explore the use of a plant derived solvent, STEPOSOL ${ }^{\circledR}$ MET-10U, ${ }^{15}$ otherwise known as $\mathrm{N}, \mathrm{N}$-dimethyl-9decenamide (Figure 1), for the synthesis of a diverse series of MOFs. Produced by Stepan ${ }^{\circledR}$ in collaboration with Elevance Renewable Sciences ${ }^{\circledR}$, STEPOSOL ${ }^{\circledR}$ MET-10U is synthesized using a biodegradable $\mathrm{C} 10$ methyl ester, generated by the cross metathesis of plant oils, such as canola, soybean and palm oil, with olefins. ${ }^{16,17}$ The industrial olefin metathesis process is performed under low-pressure and low-temperature conditions, in the presence of a highly selective proprietary catalyst. The production of this C10 methyl ester by Elevance Renewable Sciences ${ }^{\circledR}$ is thus in line with several of the 12 principles of green chemistry including: prevention (principle \#1), less hazardous chemical synthesis (principle \#3), safer solvents and auxiliaries (principle \#5), design for energy efficiency (principle \#6), use of renewable feedstocks (principle \#7), catalysis (principle \#9), and design for degradation (principle \#10). The C10 methyl ester is then converted to $N, N$ dimethyl-9-decenamide (STEPOSOL ${ }^{\circledR}$ MET-10U), a disubstituted amide composed of an unsaturated hydrocarbon chain with a terminal alkene functionality. STEPOSOL ${ }^{\circledR}$ MET-10U, a solvent that is also recognized as a powerful surfactant, has a boiling

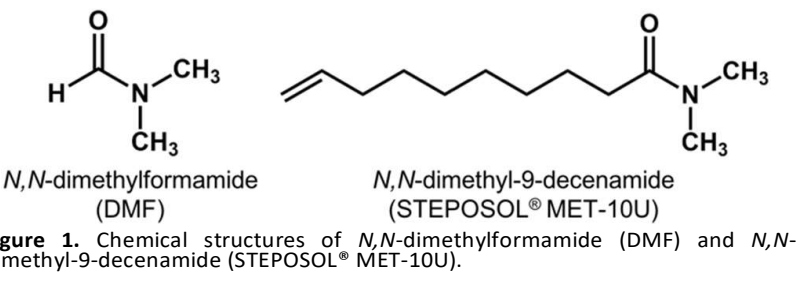


point of $297^{\circ} \mathrm{C}$, and has a biorenewable carbon index $(\mathrm{BCl})$ of $75 \%{ }^{15}$ In addition, the Hansen solubility parameter space of STEPOSOL ${ }^{\circledR}$ MET-10U encompasses that of hydrocarbons, esters, glycol ethers, and alcohols, demonstrating that it can dissolve materials with a wide range of chemical functionality. ${ }^{18}$ Finally, although the thermal decomposition pathway is not well studied, STEPOSOL ${ }^{\circledR}$ MET-10U is likely to undergo hydrolysis, leading to the formation of amine species (dimethylamine, dimethylammonium), and decanoic acid. ${ }^{19}$ The generation of low concentrations of dimethylamine is thought to aid in MOF synthesis by deprotonating the organic linker and facilitating slow and dynamic coordination between the metal and linker precursors. ${ }^{20}$ Furthermore, the generation of a carboxylic acid (such as decanoic acid, or formic acid in the case of DMF) can aid in MOF synthesis by acting as a modulator and/or stabilizing capping ligand. ${ }^{21}$ Given the high boiling point, broad Hansen solubility parameter space, potential to generate fruitful degradation products, and bioderived nature, STEPOSOL ${ }^{\circledR}$ MET$10 U$ is well-poised to replace DMF as a greener alternative for the synthesis of MOFs. ${ }^{22}$

\section{Results and discussion}

To assess the viability of using STEPOSOL ${ }^{\circledR}$ MET-10U as a replacement for DMF in the synthesis of MOFs, we chose a small series of well-known MOFs with varying structural features, including MOF-808, ${ }^{23} \mathrm{NU}-1000,{ }^{24} \mathrm{HKUST}-1,{ }^{25}$ and ZIF-8. ${ }^{26} \mathrm{MOF}$ 808 is a $\mathrm{Zr}_{6}$-based MOF with open metal sites comprised of 6 connected $\mathrm{Zr}_{6}$-cluster nodes bridged by tritopic 1,3,5benzetricarboxylic acid (BTC) linkers giving rise to a framework with spn topology (Figure 2). ${ }^{23} \mathrm{NU}-1000$ is also a $\mathrm{Zr}_{6}$-based MOF but is comprised of 8-connected $\mathrm{Zr}_{6}$-cluster nodes bridged by tetratopic pyrene-based linkers, 1,3,6,8-tetrakis( $p$-benzoic acid) pyrene ( $\mathrm{H}_{4}$ TBAPy), with an overall csq topology (Figure 2$) .{ }^{24}$ HKUST-1 is a $\mathrm{Cu}_{2}$-based MOF comprised of 4-connected $\mathrm{Cu}_{2-}$ cluster nodes bridged by BTC linkers giving a tbo topology (Figure 2). ${ }^{25}$ Finally, ZIF-8 is a Zn-based MOF comprised of Zn ion metal nodes, bridged by 2-methylimidazole $(2-\mathrm{m} / \mathrm{m})$ linkers with sod topology (Figure 2). ${ }^{26}$ This diverse series of MOFs represents frameworks with (i) ditopic, tritopic, and tetratopic organic linkers, (ii) divalent and tetravalent metals, (ii) metal ion and cluster nodes with varying connectivity, and (iv) structures with channel-type and cage-type pore architectures. In all cases, STEPOSOL ${ }^{\circledR}$ MET-10U could be used directly in place of DMF with reaction conditions nearly identical to those of reported solvothermal procedures $11 \mathrm{~b}, 23,27$ pertaining to reaction precursors, time, concentration, and temperature, without optimization.

The bulk crystallinity and the phase purity of the MOFs synthesized in STEPOSOL ${ }^{\circledR}$ MET-10U was confirmed by powder $X$-ray diffraction (PXRD) (Figure 3, Figures S1-S2) showing that each MOF was successfully synthesized. In the case of ZIF-8, single crystals suitable for X-ray diffraction were obtained without any synthetic optimization required, further highlighting the utility of STEPOSOL ${ }^{\circledR}$ MET-10U for obtaining high quality samples.

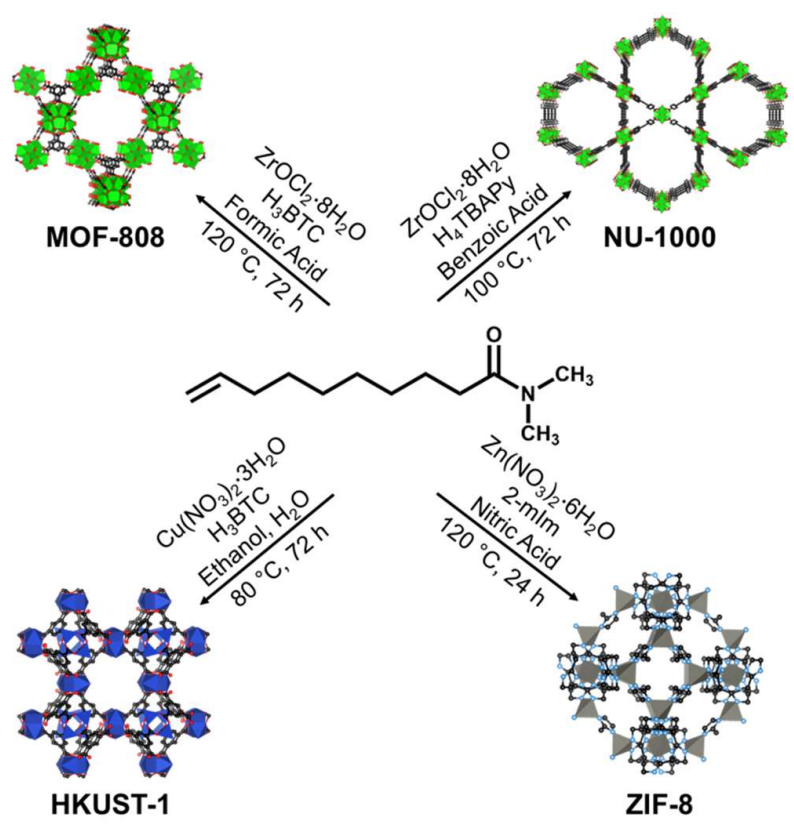

Figure 2. MOFs successfully synthesized with STEPUSOL ${ }^{*}$ MET-1OU, including MOF-808, NU-1000, HKUST-1, and ZIF-8.

The surface area and porosity of the MOFs synthesized in STEPOSOL ${ }^{\circledR}$ MET-10U was determined and compared to values obtained for materials synthesized using standard solvothermal procedures in DMF. The MOFs were characterized using $\mathrm{N}_{2}$ adsorption-desorption analysis performed at $77 \mathrm{~K}$, where each activated MOF demonstrates the expected, reversible isotherm. This includes Type I(b) for MOF-808, Type IV(b) for NU-1000, and Type I(a) for both HKUST-1 and ZIF-8 (Figure 4). Moreover, the MOFs exhibit Brunauer-Emmett-Teller (BET) areas that are comparable to those obtained for the MOFs synthesized with DMF, in our hands, using identical procedures and reagents $11 \mathrm{~b}$, 23, 27b, 28 with values of 1090 (1310), 1635 (1865), 1230 (1645), and 670 (1435) $\mathrm{m}^{2} \mathrm{~g}^{-1}$ for MOF-808, NU-1000, HKUST-1, and ZIF8 , synthesized in STEPOSOL ${ }^{\circledR}$ MET-10U and (DMF), respectively (Figures S3-S5). Pore size distribution analysis calculated using non-local density functional theory (NLDFT) for the MOFs synthesized with STEPOSOL ${ }^{\circledR}$ MET-10U, show the expected pore diameters of $18.4 \AA$, $29.5 \AA$, $12.0 \AA$, and $15.2 \AA$, for MOF-808, NU-1000, HKUST-1, and ZIF-8 respectively (Figure S6). Although synthetic protocols using STEPOSOL ${ }^{\circledR}$ MET-10U did not need to be modified, the solvent exchange procedure performed prior to MOF activation under heat and vacuum required optimization to obtain surface areas comparable to those previously reported. An array of organic solvents that are miscible with STEPOSOL ${ }^{\circledR}$ MET-10U, and more environmentally friendly than $\mathrm{DMF}^{29}$ were tested for solvent exchange, including toluene, methanol, ethanol, ethyl acetate and ethyl lactate. The highest surface areas were obtained when washing with toluene for MOF-808, methanol for HKUST-1, and ethanol 95\% for ZIF-8 (Figure S4). Therefore, in these examples, DMF was replaced with a greener alternative in both the synthesis and solvent exchange processes. Solvent exchange and activation of NU-1000, however, was most successful using 
(a)

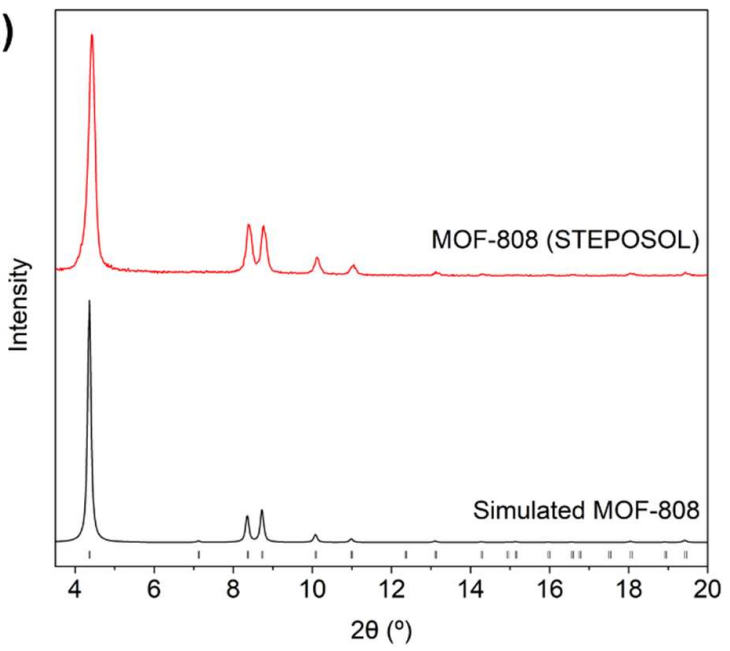

(c)

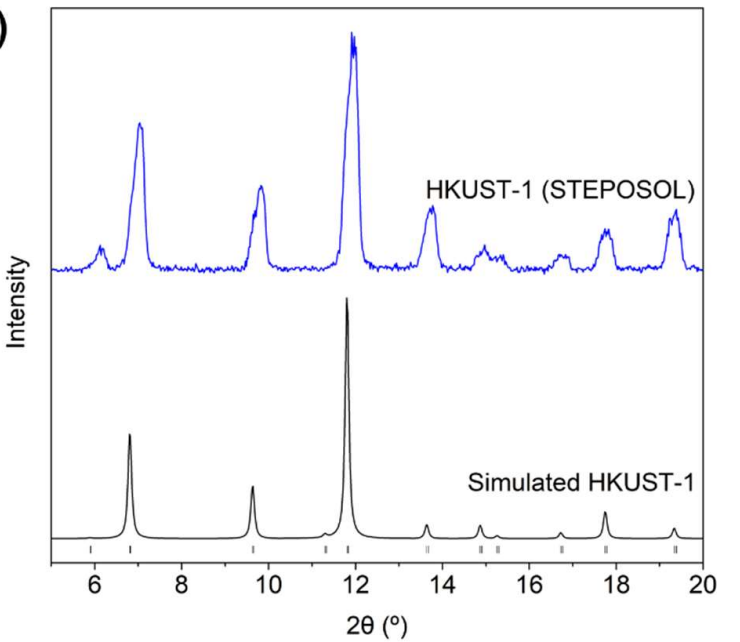

(b)

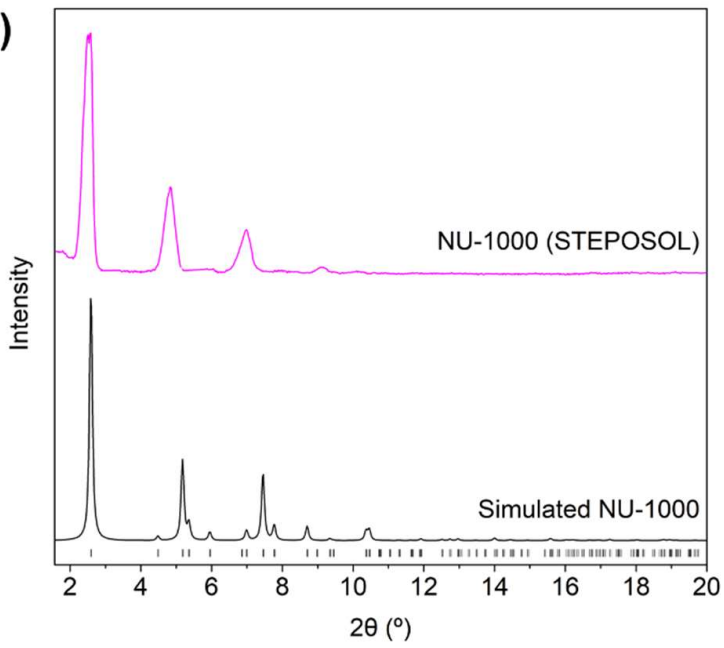

(d)

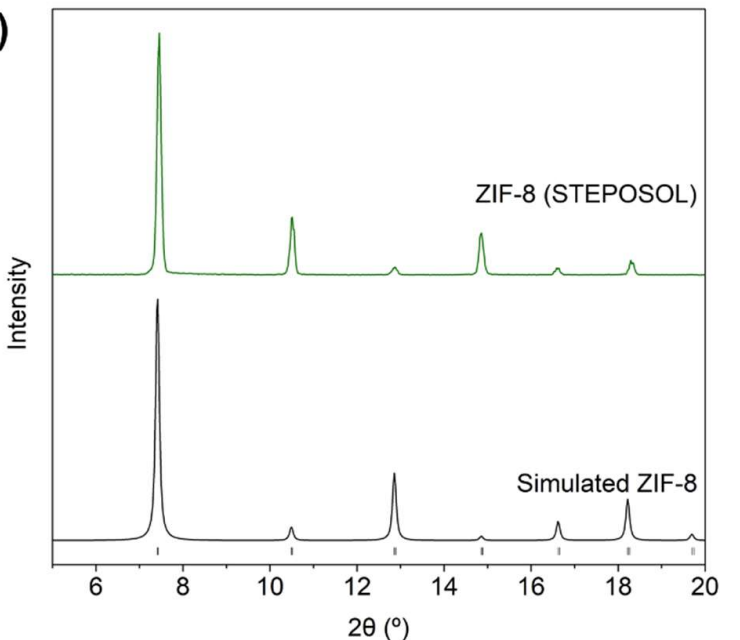

Figure 3. Simulated and experimental PXRD patterns of (a) MOF-808 synthesized by STEPOSOL ${ }^{\oplus}$ MET-10U, (b) NU-1000 synthesized by STEPOSOL ${ }^{\circledR}$ MET-10U (simulated pattern of NU-1000 (FIFFUX) was recorded at 100 K), (c) HKUST-1 synthesized by STEPOSOL ${ }^{\circledast}$ MET-10U, and (d) ZIF-8 synthesized by STEPOSOL ${ }^{\oplus}$ MET-10U.

DMF (Figure S3b), meaning that the amount of DMF used to synthesize and activate NU-1000 was minimized, but could not be eliminated entirely from the process.

In order to evaluate the thermal stability of the activated MOFs synthesized with STEPOSOL ${ }^{\circledR}$ MET-10U, and compare to those synthesized in DMF, we performed thermogravimetric analysis (TGA). In all cases, the thermogram was in excellent agreement with those obtained for the MOFs synthesized in DMF (Figure S7). The MOFs were further characterized using diffuse reflectance infrared Fourier transform spectroscopy (DRIFTS) to gain information about the infrared active functional groups in the materials. The DRIFTS data demonstrates the expected absorption bands corresponding to carboxylate (MOF-808, NU-1000, and HKUST-1) and methylimidazolate (ZIF-8) linker stretching (Figure S8), as well as $\mathrm{O}-\mathrm{H}$ stretching bands corresponding to the terminal $-\mathrm{OH}$ ligands in the node of MOF-808 and NU-1000 (Figure S8). Proton nuclear magnetic resonance ( $\left.{ }^{1} \mathrm{H}-\mathrm{NMR}\right)$ spectroscopy of the digested MOF samples synthesized in STEPOSOL ${ }^{\circledR}$ MET-10U shows the linker purity and incorporation into the MOF

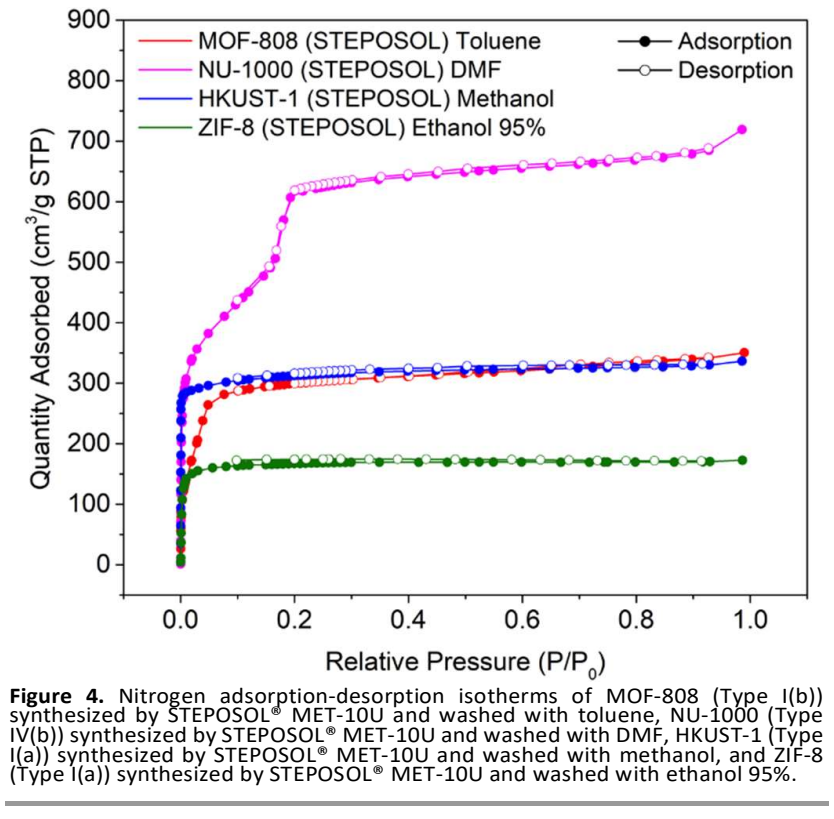


structures, with only trace amounts of STEPOSOL ${ }^{\circledR}$ MET-10U leftover post-activation (Figures S9-S13). Finally, in order to further explore the samples synthesized with STEPOSOL ${ }^{\circledR}$ MET$10 \mathrm{U}$, and compare to those synthesized by DMF, scanning electron microscopy (SEM) images were captured to confirm the morphology of the MOF crystallites. In each case the expected morphology was demonstrated. Specifically, octahedral microcrystallites for MOF-808 ( 1.5 $\mu \mathrm{m})$, hexagonal rods with rectangular facets for NU-1000 ( 100 $\mu \mathrm{m})$, octahedral microcrystallites for HKUST-1 ( 1 $\mu \mathrm{m})$, and a mixture of hexagonal-faceted crystals, cubic crystals, and rhombic dodecahedrons for ZIF-8 ( 150 $\mu \mathrm{m}$ ) (Figures S14-S18).

\section{Conclusions}

The use of green solvent alternatives to DMF for the synthesis of MOFs, particularly where synthetic conditions do not have to be optimized significantly from those reported in DMF, is highly desired and an integral part of the green chemistry toolbox. The effectiveness of STEPOSOL ${ }^{\circledR}$ MET-10U, a bioderived solvent, was assessed for the synthesis of four structurally diverse MOFs, MOF-808, NU-1000, HKUST-1 and ZIF-8, and proved to be successful in each case. Furthermore, the MOFs synthesized with STEPOSOL ${ }^{\circledR}$ MET-10U demonstrate crystallinity, surface area and porosity, morphology, and thermal stability comparable to those synthesized using standard procedures in DMF. The use of STEPOSOL ${ }^{\circledR}$ MET-10U for MOF synthesis holds significant promise, and allows for less hazardous chemical synthesis, as well as the use of renewable feedstocks and safer solvents, which is of utmost importance in both academic industry settings where the synthesis and study of MOFs is flourishing.

\section{Author Contributions}

CRediT authorship contribution statement. Paola Marino: Conceptualization, Methodology, Validation, Formal analysis, Investigation, Writing - Original Draft, Writing - Review \& Editing, Visualization. P. Rafael Donnarumma: Investigation. Hudson de Aguiar Bicalho: Investigation. Victor Quezada-Novoa: Resources. Hatem M. Titi: Formal analysis, Investigation. Ashlee J. Howarth: Conceptualization, Methodology, Resources, Writing - Review \& Editing, Supervision, Funding acquisition.

\section{Conflicts of interest}

There are no conflicts to declare.

\section{Acknowledgements}

P.M. thanks the Sustainability Action Fund of Concordia University for the Sustainability Research Award, the Howarth and Majewski research group members for offering a supportive research environment, and Prof. Marek B. Majewski for his insight and encouragement. We are grateful to Prof. Tomislav Friščić for providing access to X-ray diffraction instrumentation. We acknowledge the support of the Natural Sciences and Engineering Research Council of Canada (NSERC), [funding reference number: DGECR-2018-00344]. Cette recherche a été financée par le Conseil de recherches en sciences naturelles et en génie du Canada (CRSNG), [numéro de reference: DGECR-2018-00344].

\section{Notes and references}

1 (a) P. T. Anastas and J. C. Warner, Green Chemistry: Theory and Practice, Oxford University Press, 1998; (b) R. A. Sheldon, Chem. Soc. Rev., 2012, 41, 1437-1451; (c) P. Anastas and N. Eghbali, Chem. Soc. Rev., 2010, 39, 301-312; (d) H. C. Erythropel, J. B. Zimmerman, T. M. de Winter, L. Petitjean, F. Melnikov, C. H. Lam, A. W. Lounsbury, K. E. Mellor, N. Z. Janković, Q. Tu, L. N. Pincus, M. M. Falinski, W. Shi, P. Coish, D. L. Plata and P. T. Anastas, Green Chem., 2018, 20, 1929-1961; (e) P. T. Anastas and J. B. Zimmerman, Green Chem., 2019, 21, 6545-6566.

2 P. D. Tortell, Proc. Natl. Acad. Sci., 2020, 117, 8683-8691.

3 (a) Y. Kinoshita, I. Matsubara, T. Higuchi and Y. Saito, Bull. Chem. Soc. Jpn., 1959, 32, 1221-1226; (b) B. F. Hoskins and R. Robson, J. Am. Chem. Soc., 1989, 111, 5962-5964; (c) O. M. Yaghi and H. Li, J. Am. Chem. Soc., 1995, 117, 10401-10402; (d) O. M. Yaghi, G. Li and H. Li, Nature, 1995, 378, 703-706; (e) M. Kondo, T. Yoshitomi, H. Matsuzaka, S. Kitagawa and K. Seki, Angew. Chem. Int. Ed. Engl., 1997, 36, 1725-1727; (f) A. K. Cheetham, G. Férey and T. Loiseau, Angew. Chem. Int. Ed., 1999, 38, 3268-3292; (g) H. Li, M. Eddaoudi, M. O'Keeffe and O. M. Yaghi, Nature, 1999, 402, 276-279.

4 (a) S. Ma and H.-C. Zhou, J. Am. Chem. Soc., 2006, 128, 1173411735; (b) J. A. Mason, M. Veenstra and J. R. Long, Chem. Sci., 2014, 5, 32-51; (c) D.-X. Xue, Y. Belmabkhout, O. Shekhah, H. Jiang, K. Adil, A. J. Cairns and M. Eddaoudi, J. Am. Chem. Soc., 2015, 137, 5034-5040; (d) M. Ding, R. W. Flaig, H. L. Jiang and O. M. Yaghi, Chem. Soc. Rev., 2019, 48, 2783-2828.

5 (a) J. Lee, O. K. Farha, J. Roberts, K. A. Scheidt, S. T. Nguyen and J. T. Hupp, Chem. Soc. Rev., 2009, 38, 1450-1459; (b) P. Li, R. C. Klet, S. Y. Moon, T. C. Wang, P. Deria, A. W. Peters, B. M. Klahr, H. J. Park, S. S. Al-Juaid, J. T. Hupp and O. K. Farha, Chem. Commun., 2015, 51, 10925-10928; (c) M. Rimoldi, A. J. Howarth, M. R. DeStefano, L. Lin, S. Goswami, P. Li, J. T. Hupp and O. K. Farha, ACS Catal., 2016, 7, 997-1014; (d) K.-i. Otake, Y. Cui, C. T. Buru, Z. Li, J. T. Hupp and O. K. Farha, J. Am. Chem. Soc., 2018, 140, 8652-8656; (e) C. Wang, B. An and W. Lin, ACS Catal., 2019, 9, 130-146; (f) D. Yang and B. C. Gates, ACS Catal., 2019, 9, 17791798; $(g)$ V. Quezada-Novoa, H. M. Titi, A. A. Sarjeant and A. J. Howarth, ChemRxiv, 2020, DOI: 10.26434/chemrxiv.12355406.

6 (a) C. A. Bauer, T. V. Timofeeva, T. B. Settersten, B. D. Patterson, V. H. Liu, B. A. Simmons and M. D. Allendorf, J. Am. Chem. Soc., 2007, 129, 7136-7144; (b) L. E. Kreno, K. Leong, O. K. Farha, M. Allendorf, R. P. Van Duyne and J. T. Hupp, Chem. Rev., 2012, 112, 1105-1125.

7 (a) A. J. Howarth, Y. Liu, J. T. Hupp and O. K. Farha, CrystEngComm, 2015, 17, 7245-7253; (b) P. A. Kobielska, A. J. Howarth, O. K. Farha and S. Nayak, Coord. Chem. Rev., 2018, 358, 92-107; (c) M. Mon, R. Bruno, J. Ferrando-Soria, D. Armentano and E. Pardo, J. Mater. Chem. A, 2018, 6, 4912-4947; (d) R. J. Drout, L. Robison, Z. Chen, T. Islamoglu and O. K. Farha, Trends Chem., 2019, 1, 304-317.

8 (a) P. Horcajada, C. Serre, G. Maurin, N. A. Ramsahye, F. Balas, M. Vallet-Regí, M. Sebban, F. Taulelle and G. Férey, J. Am. Chem. 
Soc., 2008, 130, 6774-6780; (b)C. Orellana-Tavra, R. J. Marshall, E. F. Baxter, I. A. Lázaro, A. Tao, A. K. Cheetham, R. S. Forgan and D. Fairen-Jimenez, J. Mater. Chem. B, 2016, 4, 7697-7707.

9 (a) M. B. Majewski, A. W. Peters, M. R. Wasielewski, J. T. Hupp and O. K. Farha, ACS Energy Lett., 2018, 3, 598-611; (b) Y. Isaka, Y. Kondo, Y. Kawase, Y. Kuwahara, K. Mori and H. Yamashita, Chem. Commun., 2018, 54, 9270-9273.

10 (a) T. Faust, Nat. Chem., 2016, 8, 990-991; (b) Nat. Chem., 2016, 8, 987-987.

11 (a) K. Užarević, T. C. Wang, S.-Y. Moon, A. M. Fidelli, J. T. Hupp, O. K. Farha and T. Friščić, Chem. Commun., 2016, 52, 2133-2136; (b) J. Zhang, G. B. White, M. D. Ryan, A. J. Hunt and M. J. Katz, ACS Sustain. Chem. Eng., 2016, 4, 7186-7192; (c) P. A. Julien, C. Mottillo and T. Friščić, Green Chem., 2017, 19, 2729-2747; (d) H. M. Titi, J.-L. Do, A. J. Howarth, K. Nagapudi and T. Friščić, Chem. Sci., 2020, 11, 7578-7584.

12 (a) Z. Ajoyan, P. Marino and A. J. Howarth, CrystEngComm, 2018, 20, 5899-5912; (b) S. Kumar, S. Jain, M. Nehra, N. Dilbaghi, G. Marrazza and K.-H. Kim, Coord. Chem. Rev., 2020, 420, 213407.

13 Preventing Adverse Health Effects from Exposure to: Dimethylformamide (DMF),

https://www.cdc.gov/niosh/docs/90-105/default.html, (accessed March 22, 2021).

14 F. P. Byrne, S. Jin, G. Paggiola, T. H. M. Petchey, J. H. Clark, T. J. Farmer, A. J. Hunt, C. Robert McElroy and J. Sherwood, Sustain. Chem. Process., 2016, 4, 1-24.

15 STEPOSOL ${ }^{\circledR}$ MET-10U, https://www.stepan.com/content/stepan-dotcom/en/products-markets/product/STEPOSOLMET10U.html, (accessed March 22, 2021).

16 Stepan introduces STEPOSOL ${ }^{\circledR}$ MET-10U as part of its joint development agreement with Elevance, https://elevance.com/news/stepan-introduces-steposol-met10u-as-part-of-its-joint-development-agreement-withelevance/, (accessed March 22, 2021).

17 Sustainability, https://elevance.com/sustainability/, (accessed March 22, 2021)

18 Stepan $^{\circledR}$, STEPOSOL ${ }^{\circledR}$ MET-10U Product Bulletin, 2019.

19 (a) G. Para, J. Łuczyński, J. Palus, E. Jarek, K. A. Wilk and P. Warszyński, J. Colloid Interface Sci., 2016, 465, 174-182; (b) A. D. Burrows, K. Cassar, R. M. W. Friend, M. F. Mahon, S. P. Rigby and J. E. Warren, CrystEngComm, 2005, 7, 548-550; (c) N. Stock and S. Biswas, Chem. Rev., 2012, 112, 933-969.

20 A. J. Howarth, A. W. Peters, N. A. Vermeulen, T. C. Wang, J. T. Hupp and O. K. Farha, Chem. Mater., 2016, 29, 26-39.

21 C. V. McGuire and R. S. Forgan, Chem. Commun., 2015, 51, 51995217.

22 D. Morelli Venturi, F. Campana, F. Marmottini, F. Costantino and L. Vaccaro, ACS Sustain. Chem. Eng., 2020, 8, 17154-17164.

23 H. Furukawa, F. Gandara, Y. B. Zhang, J. Jiang, W. L. Queen, M. R. Hudson and O. M. Yaghi, J. Am. Chem. Soc., 2014, 136, 43694381.

24 J. E. Mondloch, W. Bury, D. Fairen-Jimenez, S. Kwon, E. J. DeMarco, M. H. Weston, A. A. Sarjeant, S. T. Nguyen, P. C. Stair, R. Q. Snurr, O. K. Farha and J. T. Hupp, J. Am. Chem. Soc., 2013, 135, 10294-10297.

25 S. S. Y. Chui, S. M. F. Lo, J. P. H. Charmant, A. G. Orpen and I. D. Williams, Science, 1999, 283, 1148.

26 K. S. Park, Z. Ni, A. P. Côté, J. Y. Choi, R. Huang, F. J. Uribe-Romo, H. K. Chae, M. O'Keeffe and O. M. Yaghi, Proc. Natl. Acad. Sci., 2006, 103, 10186.
27 (a) T. C. Wang, N. A. Vermeulen, I. S. Kim, A. B. Martinson, J. F. Stoddart, J. T. Hupp and O. K. Farha, Nat. Protoc., 2016, 11, 149162; (b) O. Karagiaridi, M. B. Lalonde, W. Bury, A. A. Sarjeant, O. K. Farha and J. T. Hupp, J. Am. Chem. Soc., 2012, 134, 1879018796.

28 T. Islamoglu, K.-i. Otake, P. Li, C. T. Buru, A. W. Peters, I. Akpinar, S. J. Garibay and O. K. Farha, CrystEngComm, 2018, 20, 59135918.

29 Methanol, Ethanol and Ethyl Acetate are listed as "preferred" solvents by Pfizer, whereas Toluene is listed as "usable" by Pfizer and "Substitution Advisable" by Sanofi. (Ref 14)
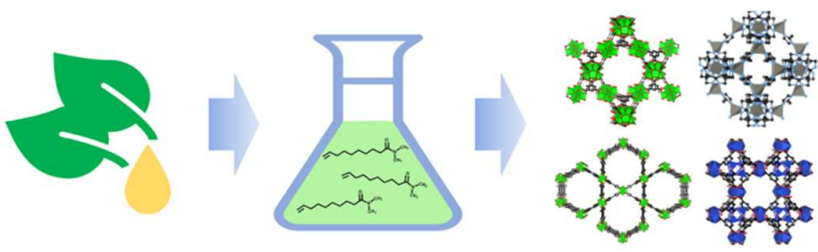

TOC Graphic 